\title{
Radiation- and anthracycline-induced cardiac toxicity and the influence of ErbB2 blocking agents
}

\author{
Ingar Seemann · Johannes A. M. te Poele • \\ Ji-Ying Song $\cdot$ Saske Hoving $\cdot$ Nicola S. Russell • \\ Fiona A. Stewart
}

Received: 19 July 2013/Accepted: 18 September 2013/Published online: 4 October 2013

(C) The Author(s) 2013. This article is published with open access at Springerlink.com

\begin{abstract}
In Her2-positive breast cancer patients, inhibition of epidermal growth factor receptor 2 (ErbB2)signaling is often combined with chemotherapy and radiotherapy. The risk of cardiac toxicity after anthracyclines and radiotherapy is recognized, but little is known about increased risk when these treatments are combined with ErbB2 inhibition. This study investigated whether ErbB2 inhibition increased radiation or anthracyclineinduced toxicity. In an in vitro study, human cardiomyocytes were treated with irradiation or doxorubicin, alone or in combination with trastuzumab, and evaluated for cell survival and growth. Groups of mice received 0 or $14 \mathrm{~Gy}$ to the heart, alone or in combination with lapatinib, or $3 \times 4 \mathrm{mg} / \mathrm{kg}$ doxorubicin alone or in combination with lapatinib. Mice were evaluated 40 weeks after treatment for cardiac damage. Changes in cardiac function $\left({ }^{99 \mathrm{~m}} \mathrm{Tc}-\right.$ Myoview gated SPECT) were related to histomorphology and microvascular damage. Radiation or doxorubicininduced cardiomyocyte toxicity (in vitro) were not exacerbated by trastuzumab. Cardiac irradiation of mice
\end{abstract}

Electronic supplementary material The online version of this article (doi:10.1007/s10549-013-2707-7) contains supplementary material, which is available to authorized users.

I. Seemann - J. A. M. te Poele · S. Hoving · F. A. Stewart ( $\square)$ Division of Biological Stress Response (H3), The Netherlands Cancer Institute, Plesmanlaan 121, 1066 CX Amsterdam, The Netherlands

e-mail: f.stewart@nki.nl

J.-Y. Song

Department of Experimental Animal Pathology, The

Netherlands Cancer Institute, Amsterdam, The Netherlands

N. S. Russell

Division of Radiotherapy, The Netherlands Cancer Institute,

Amsterdam, The Netherlands decreased microvascular density (MVD) and increased endothelial damage in surviving capillaries (decrease alkaline phosphatase expression and increased von Willebrand factor), but these changes were not exacerbated by lapatinib. Inflammatory responses in the irradiated epicardium (CD45+ and F4/80+ cells) were significantly reduced in combination with lapatinib. Irradiation, doxorubicin, and lapatinib each induced cardiac fibrosis but this was not further enhanced when treatments were combined. At the ultra-structural level, both lapatinib and doxorubicin induced mitochondrial damage, which was enhanced in combined treatments. Lapatinib alone also induced mild changes in cardiac function but this was not enhanced in the combined treatments. Trastuzumab did not enhance direct radiation or anthracycline toxicity of cardiomyocytes in vitro. Lapatinib did not enhance the risk of radiation or anthracycline-induced cardiac toxicity in mice up to 40 weeks after treatment, but mitochondrial damage was more severe after doxorubicin combined with lapatinib.

Keywords ErbB2 - Cardiac microvasculature . Irradiation · Anthracyclines

\section{Introduction}

Cardiovascular damage has been reported as a long-term toxicity in breast cancer survivors previously treated with radiotherapy (RT) and anthracycline chemotherapy (CT) [1]. Prospective functional imaging studies show that $\sim 40 \%$ of left-sided breast cancer patients treated with RT develop asymptomatic cardiac perfusion defects within 2 years and about $16 \%$ eventually develop wall-motion abnormalities [2-4]. Yearly, 1.5 million new breast cancer 
patients are diagnosed worldwide who will undergo RT, CT, surgery, and/or adjuvant hormonal therapy. Approximately $15-20 \%$ of breast cancers show a highly aggressive subtype, characterized by epidermal growth factor receptor 2 (ErbB2) overexpression. Such cancers are fast-growing, highly invasive, resistant to CT and RT and are therefore associated with higher risk for recurrence [5, 6]. Epidermal growth factor receptor 1 (ErbB1) is also expressed in several human tumors, including breast cancer, and makes significant contributions to invasion and growth of tumors [7].

The established role of ErbB1 and ErbB2 in breast cancer makes them attractive therapeutic targets. Trastuzumab, a humanized anti-ErbB2 monoclonal antibody, is the most prominent, first-line agent for ErbB2 (HER2)overexpressing metastatic breast cancer [8]. Clinical studies using trastuzumab confirmed the benefits of inhibiting ErbB2 signaling, including inhibition of $\mathrm{p} 27^{\mathrm{Kip} 1}$, activation of PTEN tumor suppressor gene and induction of G1 cell cycle arrest [9]. Adjuvant treatment with trastuzumab for operable breast cancer improves overall survival rate in Her2-positive breast cancer patients by $\sim 30 \%$ and reduces the risk of recurrence by $\sim 50 \%[10,11]$. However, heart failure occurs in $1.7-4.1 \%$ of patients treated with trastuzumab and cardiac toxicity lead to discontinuation of the adjuvant treatment in $19 \%$ of the patients [10-12].

ErbB2 is an orphan receptor that has no ligand binding site but dimerizes with other ligand-bound EGRF receptors (HER3, HER4). One of the most common ligands of the EGFR pathway in the heart is neuregulin-1. Targeting both ErbB1 and ErbB2 is hypothesized to have superior therapeutic effects relative to single-agent treatment. Dual inhibitor lapatinib (GW572016) is a small molecule, reversible inhibitor of the tyrosine kinase activities of ErbB1 and ErbB2 at equal potency. Lapatinib works by blocking the signaling transduction to Ras/Raf MAPKs and the PI3K/Akt pathway, which leads to increased apoptosis and decreased cellular proliferation. Perez and colleagues reviewed 44 studies in which lapatinib (as monotherapy or in combination with previously given anthracyclines or trastuzumab) induced low levels of cardiac toxicity, as detected by reversible decreased left ventricle ejection fraction (LVEF) [13].

The mechanisms whereby cardiac toxicity occurs after ErbB2 inhibition is not fully understood, since nonmalignant cells do not over-express ErbB2. However, ErbB2 signaling and the ligand Neuregulin-1 are known to play a crucial role in survival and growth of cardiac myocytes [14, 15]. Moreover, a recent study demonstrated that irradiation inhibited ErbB2 signaling in rat hearts until the onset of fibrosis after 10 weeks. As fibrosis progressed, ErbB2 and the EGFR ligand neuregulin were significantly upregulated, presumably as an attempt to regenerate the myocardium [16]. This raises the question whether delayed inhibition of ErbB2 after CT or RT could lead to increased cardiac toxicity.

Little is known about the long-term cardiac outcome of lapatinib in combination with anthracycline CT or irradiation. In this study, we first investigated whether blocking of ErbB2 enhanced the toxicity of radiation- or doxorubicin (Dox)-treated cardiomyocytes in vitro. We subsequently investigated the influence of combined ErbB1/2 inhibition in mice treated with cardiac irradiation or systemic Dox. For these studies lapatinib was given for 20 weeks in the chow, either at the time of irradiation or Dox (direct), or delayed until 20 weeks after irradiation or Dox. This was designed to mimic clinical treatment protocols and to investigate the influence of lapatinib on the short- and longterm damage repair process following irradiation or anthracyclines. Structural and functional changes were monitored at 40 weeks after treatment to determine whether ErbB1/2 inhibition caused increased cardiac damage or inhibited recovery after radiation or anthracycline treatment.

As far as we are aware, this is the first study that characterizes in detail long-term cardiac toxicity after lapatinib in combination with irradiation or Dox.

\section{Methods}

Cell culture conditions and treatment

Human cardiac myocytes (HCM) from Promocell (Heidelberg, Germany) were cultured in DMEM (Gibco ${ }^{\circledR}$, Invitrogen) supplemented with $10 \%$ fetal calf serum, $1 \%$ penicillin, and $1 \%$ streptomycin at $37{ }^{\circ} \mathrm{C}$ with $5 \% \mathrm{CO}_{2}$. The HCM express markers of early stage differentiation such as GATA-4 and sarcomeric alpha-actin and act more like progenitor cells with capacity for proliferation. For irradiation experiments, cells were seeded in 96-well plates (1,000 per well) and irradiated with $0,2.5,5$, or $10 \mathrm{~Gy}$ $\left({ }^{137} \mathrm{Cs}\right.$ irradiation, with a dose rate of $\left.0.66 \mathrm{~Gy} / \mathrm{min}\right)$ before exposure to $0,0.1,1$, or $10 \mu \mathrm{g} / \mathrm{ml}$ trastuzumab (Roche, from the Netherlands Cancer Institute pharmacy) for 14 or 21 days. Cells were then washed $(3 \times$ with PBS) to remove drugs and evaluated for cell viability. For Dox experiments, 4,000 cells per well were seeded and treated with 0 , $0.0025,0.025,0.25,2.5,25$, and $250 \mu \mathrm{g} / \mathrm{ml}$ Dox (Doxorubicin hydrochloride $2 \mathrm{mg} / \mathrm{ml} \mathrm{PCH}$, from the Netherlands Cancer Institute pharmacy) and trastuzumab $(0-10 \mu \mathrm{g} / \mathrm{ml})$ for 3 days, washed and evaluated for cell viability directly or re-incubated with trastuzumab until evaluation at 14 days. Cell viability was detected by cell counting kit- 8 (CCK-8, Sigma, Zwijndrecht, the Netherlands) following the manufacturer's protocol. 
Fig. 1 Schedule overview. Schematic representation of schedules for lapatinib given for 20 weeks in the chow, starting at the time of irradiation or doxorubicin (direct), or starting 20 weeks after irradiation or doxorubicin (delayed)

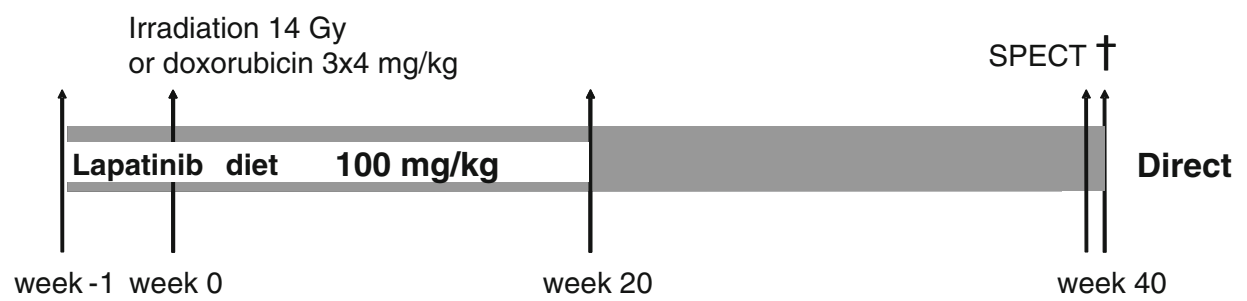

Irradiation $14 \mathrm{~Gy}$

or doxorubicin $3 \times 4 \mathrm{mg} / \mathrm{kg}$

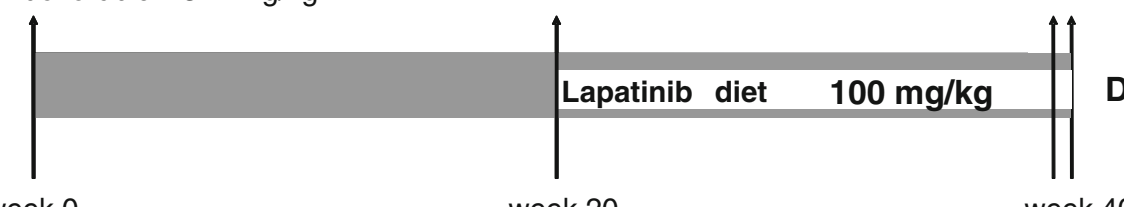

Mice and treatments groups

Male C57BL/6 mice, aged 8-12 weeks (Charles River Laboratories, France) were randomly allocated to receive 0 Gy or 14 Gy to the heart, or $4 \mathrm{mg} / \mathrm{kg}$ Dox intraperitoneal (i.p.) weekly for 3 weeks. Separate cohorts of animals were included for irradiation or anthracycline combined with lapatinib, as well as age matched controls. Lapatinib (from the Netherlands Cancer Institute pharmacy) was mixed with standard mouse chow to a final concentration of $0.48 \mathrm{~g} / \mathrm{kg}$. Assuming consumption of $5 \mathrm{~g}$ chow/mouse/day, this is equivalent to $100 \mathrm{mg} / \mathrm{kg} /$ day. A pilot study with $100 \mathrm{mg} / \mathrm{kg} /$ day given in chow or by oral gavages resulted in the same plasma concentration (mean $946 \mathrm{ng} / \mathrm{ml}$ and $909 \mathrm{ng} / \mathrm{ml}$ lapatinib after chow diet and oral gavages). Lapatinib diet was either started 7 days before irradiation or Dox (direct schedule) to achieve steady state plasma levels, or delayed until 20 weeks after treatment (Fig. 1). Irradiation was with $250 \mathrm{kV}$ X-rays, operating at $12 \mathrm{~mA}$ and filtered with $0.6-\mathrm{mm}$ Copper. The dose rate was $0.94 \mathrm{~Gy} / \mathrm{min}$, with a field size of $10.6 \times 15 \mathrm{~mm}$ (including the whole heart and up to $30 \%$ lung volume); the rest of the mouse was shielded with lead. For irradiation, unanesthetized mice were immobilized in a prone position in acrylic perspex jigs. Each treatment group comprised 10-15 mice ( $n=125$ in total). Experiments were in agreement with the Dutch law on animal experiments and welfare, and in line with the international Guide for the Care and Use of Laboratory Animals (Eighth edition).

Tissue preparation for histology

At termination of the experiment, the heart was perfused via the aortic arch (retro-grade), under lethal sodium pentobarbital anesthesia (18 mg per mouse, i.p.), with PBS (frozen sections) or PBS followed by $1 \%$ paraformaldehyde (paraffin sections). The heart was then quickly excised before freezing on dry ice or immersion in $1 \%$ paraformaldehyde.

Cross-sections were cut at the level of the mid-horizontal plane from fixed paraffin-embedded tissues $(4 \mu \mathrm{m})$ or frozen tissues $(7 \mu \mathrm{m})$.

\section{Frozen sections}

An anti-CD31 antibody (1:50, Becton\&Dickinson) was used to visualize cardiac vasculature. To determine functional changes in the microvasculature, a histochemical staining with Naphtol AS-MX/DMF and fast Blue BB salt was performed to detect endothelial cell alkaline phosphatase. Sections were also reacted with antibodies against von Willebrand Factor (vWF) (1:4000, Abcam, Cambridge, USA) as a marker of thrombotic changes. All sections were processed identically, with precisely the same incubation times for the primary and secondary antibody and diaminobenzidine (DAB) solution (Sigma, Zwijndrecht, the Netherlands).

For quantification of microvascular changes, five random fields ( $40 \times$ objective) of the heart were photographed with a CCD 2 Color Microscope system, including a Zeiss AxioCam color camera (Axiocam HRc, Zeiss, Göttingen, Germany). A computerized morphometry system (Leica Qwin V3, Leica, Rijswijk, the Netherlands) was used to quantify the MVD of CD31 positive structures. Vessels $<1.5$ or $>200 \mu \mathrm{m}^{2}$ were automatically excluded from the measurements. Photographs of whole sections stained for ALP and vWF were taken with an Aperio scanner (Scanscope-XT, Aperio technologies, Vista, USA) using $40 \times$ objective. Analyses of the percentage myocardium, excluding endocardium, positive for each marker were done with a computerized morphometry system (Leica Qwin V3). 


\section{Paraffin sections}

To determine the extent of inflammation, sections were immuno-labeled with anti-CD45 antibody (1:400, Becton \& Dickinson, Franklin lakes, USA). To detect alterations in the number of macrophages sections were stained with anti-F4/80 (1:300, AbD Serotec, Dusseldorf, Germany) and counted per left ventricle (LV) myocardial area.

All sections were processed identically; therefore, differences between the treatments are due to DAB identification of the relevant protein. Interstitial collagen was determined in the myocardium based on Sirius red staining.

Photographs of the LV wall, excluding the septum, were taken using a $40 \times$ objective (Leica DFC320). Interstitial collagen was quantified in five randomly selected areas of the subendocardium and myocardium of the LV $(40 \times$ objective) and results were expressed as percentage tissue positive for Sirius red relative to myocardial area. The number of CD45+ cells per section was counted separately in the epicard and myocard to determine the extent of inflammation. Morphometric parameters were analyzed using a computerized morphometry system (Leica Qwin V3). Transverse sections were stained with hematoxylin and eosin $(\mathrm{H} \& \mathrm{E})$ to check for alterations in the myocardium and blood vessels. A semi-quantitative scoring system was used, whereby for the blood vessels 1 indicates one mild/severe morphological event (degeneration of the coronary arterioles, dilation of capillaries in myocardium accompanied by edema in stroma or clotting-like materials in coronary vessels), 2 indicates two morphological events, and 3 indicates $>2$ severe morphological events. For the myocardium, a score of 1 indicated atrophy, 2 indicated degeneration or hypertrophy, and 3 indicated more than two events.

\section{Electron microscopy (EM)}

Tissues were fixed in Karnovsky's fixative, followed by $1 \%$ osmiumtetroxide in $0.1 \mathrm{M}$ cacodylate-buffer. After washing, pellets were stained with Ultrastain 1 (Leica, Vienna, Austria), followed by ethanol dehydration. Finally the cells were embedded in a mixture of DDSA/NMA/ Embed-812 (EMS, Hatfield, U.S.A), sectioned and stained with Ultrastain 2 (Leica, Vienna, Austria) and analyzed with a CM10 electron microscope (FEI, Eindhoven, the Netherlands).

\section{Gated SPECT/CT}

Gated single photon emission computed tomography (gSPECT) acquisitions were made with a small-animal NanoSPECT/CT (Bioscan Europe, Ltd., Paris, France). Animals were anesthetized with Hypnorm (Fentanyl
$0.26 \mathrm{mg} / \mathrm{kg} /$ Fluanisone $8.33 \mathrm{mg} / \mathrm{kg}$, VetaPharma, Ltd., Leeds, UK) and Dormicum (Midazolam, $4.17 \mathrm{mg} / \mathrm{kg}$, Roche, Woerden, the Netherlands) via i.p. injection (1:2:1 Hypnorm: $\mathrm{H}_{2} \mathrm{O}$ :Dormicum; $120 \mu \mathrm{l} /$ mouse). Myoview (GEhealthcare, Hoevelaken, the Netherlands) was labeled with 1-1.5 ml ${ }^{99 \mathrm{~m}}$ Tc-pertechnetate. For detailed information see Seemann et al. [17]. Quantitative analysis of the reconstructed datasets was performed on a clinical e.soft (syngobased) workstation (Siemens Medical Solutions, Siemens AG, Erlangen, Germany), using algorithms to automatically reconstruct a count based 3D model of the dimensions of the left ventricular (LV) end-diastolic and systolic volumes (EDV, ESV). Subsequently, a LV time volume curve and its first derivative were generated. The ejection fraction (EF) was calculated based on the difference between EDV and ESV divided by EDV and peak filling rate (PFR) (EDV/s) was calculated from the Fourier-fitted curves.

\section{Statistics}

Data are expressed as mean \pm SEM and groups were compared using non-parametric Mann-Whitney exact U-tests. Group differences were considered statistically significant at $p<0.05$. Statistical analyses were performed using SPSS version 20.

\section{Results}

HER2 inhibition did not decrease myocyte cell viability in vitro

Exposure of human cardiomyocytes for 3 days to Dox induced a dose-dependent decrease in cell viability. However, combination of Dox with trastuzumab did not further decrease myocyte cell viability (Fig. 2a). When Dox was removed after 3 days and cells were evaluated at 14 days after treatment, cell proliferation was not affected by the presence of trastuzumab. Prior exposure to $>0.025 \mu \mathrm{g} / \mathrm{ml}$ Dox inhibited cardiomyocyte growth, but this was independent of trastuzumab exposure (Fig. 2b).

Cardiomyocyte cell viability decreased after radiation doses of 2.5-10 Gy, when assessed at 14 or 21 days after treatment, but there was no further decrease in cell viability in combination with trastuzumab (Fig. 2c, d).

Mouse survival and weight

Irradiation with 14 Gy alone caused no premature deaths and few deaths occurred in combination with direct $(7 \%)$ or delayed lapatinib (12\%). Heart and body weights were slightly lower after irradiation (Fig. 3a and Table S1). There were more unscheduled deaths after treatment with Dox 
Fig. 2 Cardiomyocyte viability after ErbB2 inhibition. Fig a/b: Human cardiomyocytes were treated with doxorubicin $(0-250 \mu \mathrm{g} / \mathrm{ml})$ and trastuzumab $(0-10 \mu \mathrm{g} / \mathrm{ml})$ for 3 days. Cells were then washed $(3 \times$ with PBS) to remove drugs and evaluated for cell viability directly (2a) or re-incubated with trastuzumab only until evaluation at 14 days (2b). Each symbol represents the mean $( \pm$ SEM $)$ of three experiments and results are expressed as percentage cell survival. Fig c/d: Human cardiomyocytes were irradiated $(0,2.5,5$, or $10 \mathrm{~Gy})$ and then exposed to trastuzumab $(0-10 \mu \mathrm{g} / \mathrm{ml})$ for 14 days $(\mathbf{c})$ or 21 days (d) before evaluation of cell viability. Each symbol represents the mean $( \pm$ SEM $)$ of three experiments and results are expressed as percentage cell survival

Fig. 3 Kaplan-Meier estimated of overall survival shown for mice treated with lapatinib alone or in combination with radiation or doxorubicin.

Survival data are shown for mice treated with radiation (a) or doxorubicin (b) alone or in combination with direct or delayed with lapatinib.

Treatment groups were compared with age-matched controls. *indicates significant differences between treated mice and age matched control groups ( $p<0.05$; MannWhitney U-test)
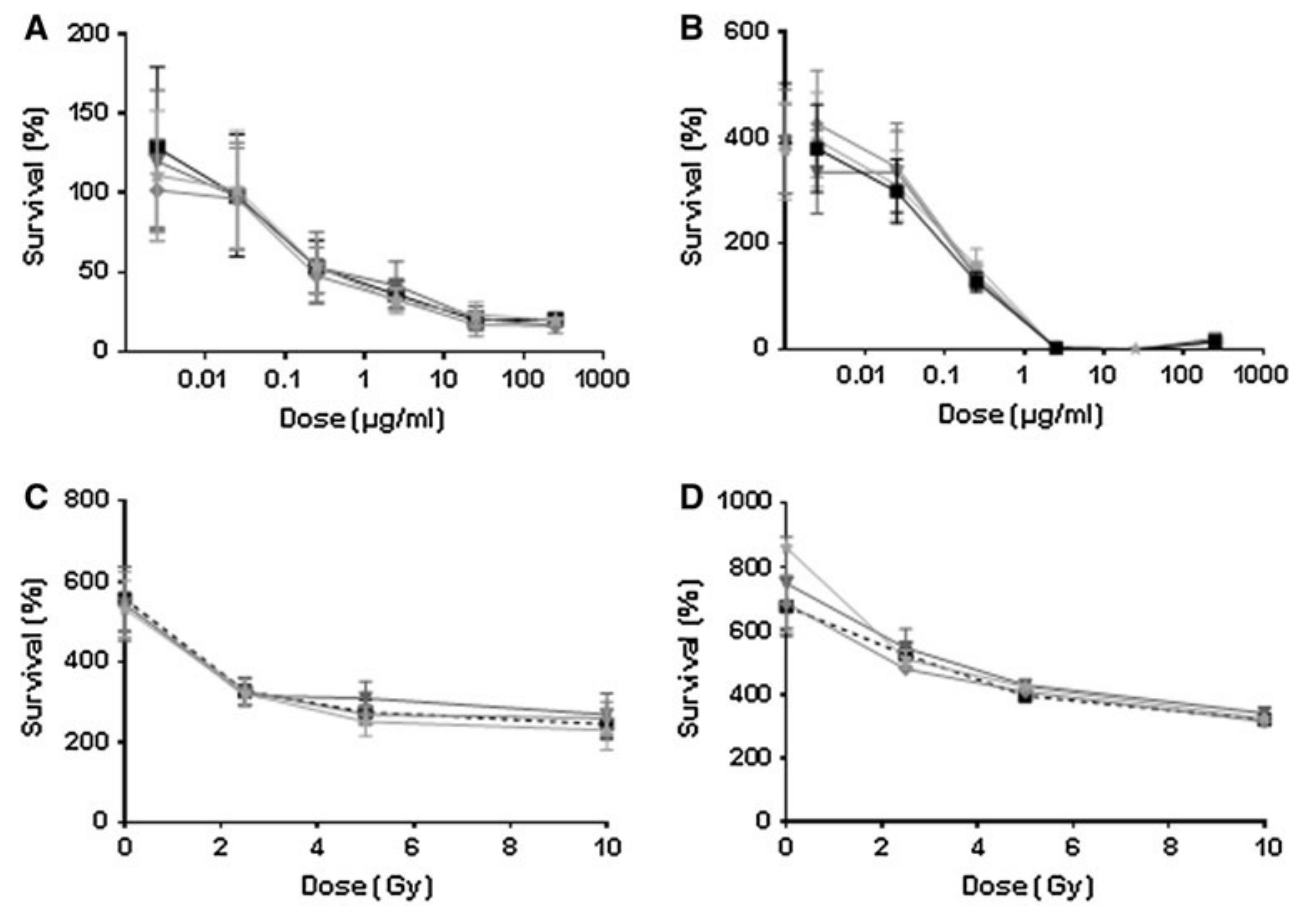
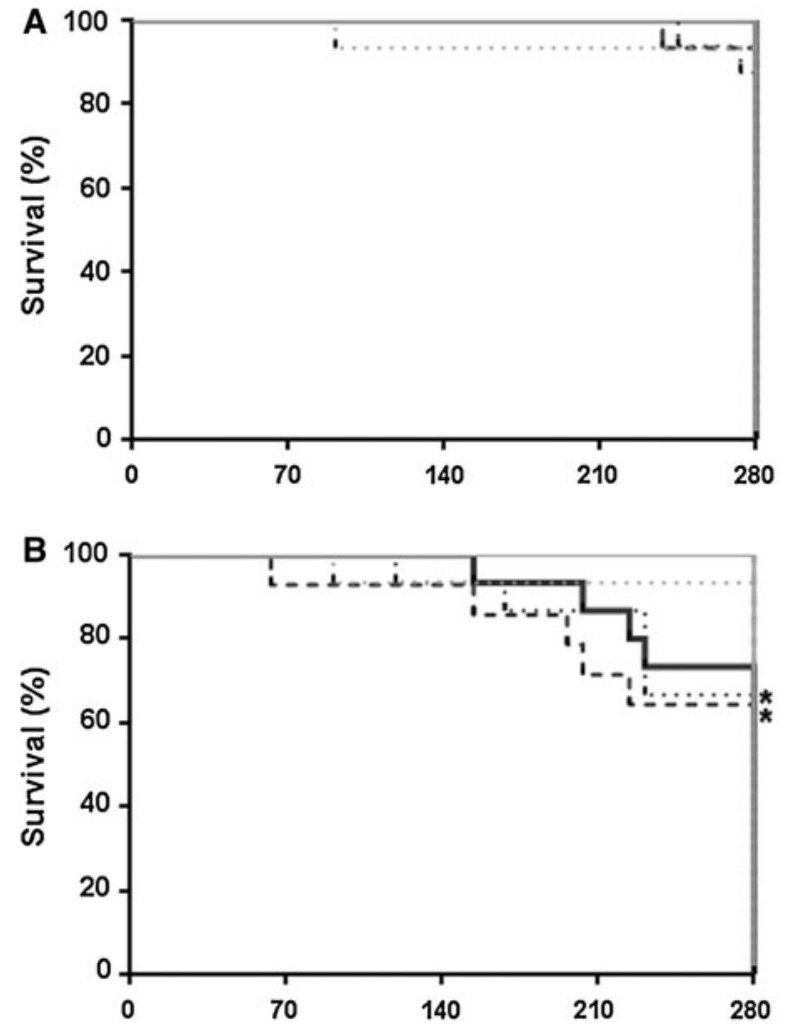

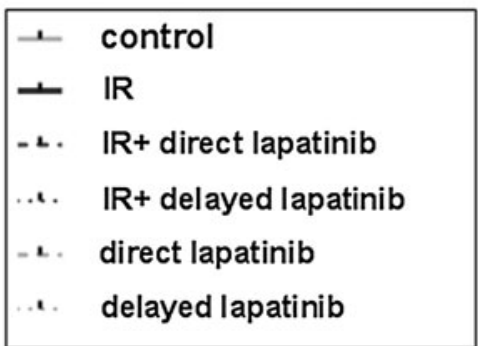


(27\%), especially when combined with lapatinib (36 and $33 \%$ deaths for direct and delayed lapatinib) (Fig. 3b; Table S2). Since the group analyses were done on material from surviving animals only, this probably represents an underestimate of the total toxic effects of Dox. Both heart and body weights were also lower after Dox alone or in combination with lapatinib compared to age-matched control.

\section{Radiation-induced microvascular damage}

MVD decreased significantly after 14 Gy radiation alone but the decline was not further enhanced by combination with either direct or delayed lapatinib treatment (Fig. 4a). There were no changes in MVD after Dox treatment alone or in combination with lapatinib (data not shown). Radiation-induced changes in MVD were accompanied by endothelial damage, as shown by a marked decrease in ALP activity (Fig. 4b) and an increased expression of the thrombotic endothelial marker vWF after irradiation alone (Fig. 4c). None of these endothelial changes were more severe after combined treatments with lapatinib then after irradiation alone (Fig. 4b, c). Endothelial damage (ALP) or thrombotic changes in the microvascular (vWF) were not detected in Dox-treated mice (data not shown).

Radiation-induced inflammation

Irradiation alone led to a significant increase in CD45+ cells and F4/80+ cells in the epicardium (Fig. 5) but not in the myocardium (data not shown). Strikingly, direct or delayed lapatinib decreased CD45+ and F4/80+ cells in the irradiated epicardium, compared to irradiation alone (Fig. 5a, b). There were no significant inflammatory responses seen in Dox-treated mice (data not shown).

Irradiation- and doxorubicin-induced cardiac fibrosis

Cardiac fibrosis was significantly increased by irradiation or lapatinib alone, when administrated directly (Fig. 6a). Lapatinib (either direct or delayed) did not further enhance the fibrosis induced by irradiation. There was also a significant increase in cardiac fibrosis after Dox alone, but this was not further enhanced by either direct or delayed lapatinib (Fig. 6b).

\section{Structural and morphological alterations}

H\&E stained sections were semi-quantitatively analyzed for changes in the myocardium and blood vessels. There were only minor changes in the vasculature after irradiation alone
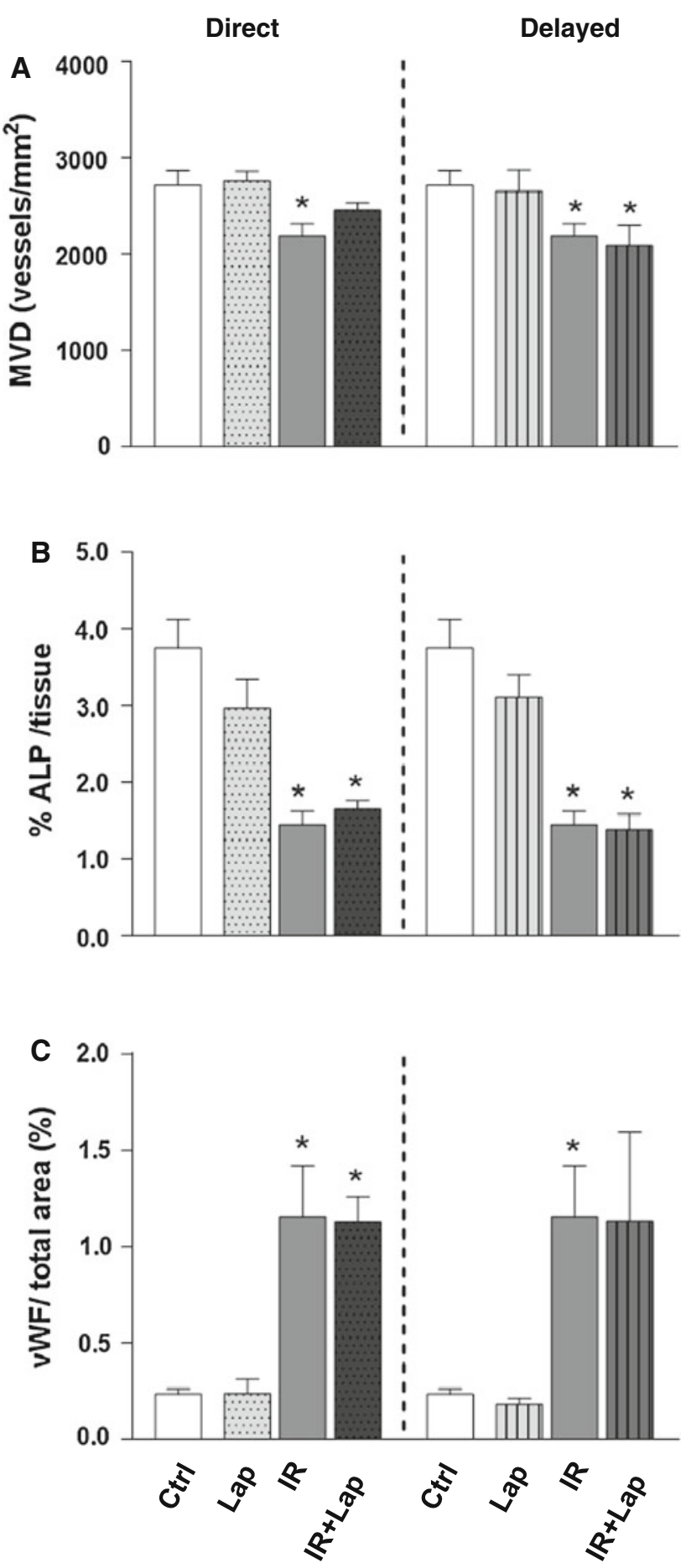

Fig. 4 Microvascular alterations after ErbB2 inhibition alone or in combination with irradiation. a MVD per unit area expressed as number of microvessels per $\mathrm{mm}^{2}$. b ALP positive tissue areas as $\%$ of total tissue. c vWF positive tissue areas as $\%$ of total tissue $* p<0.05$ compared to age-matched untreated controls. Each value represents the mean $( \pm$ SEM) for minimal five mice per group. Ctrl control; Lap lapatinib; IR irradiation; IR+lap irradiation combined with lapatinib 

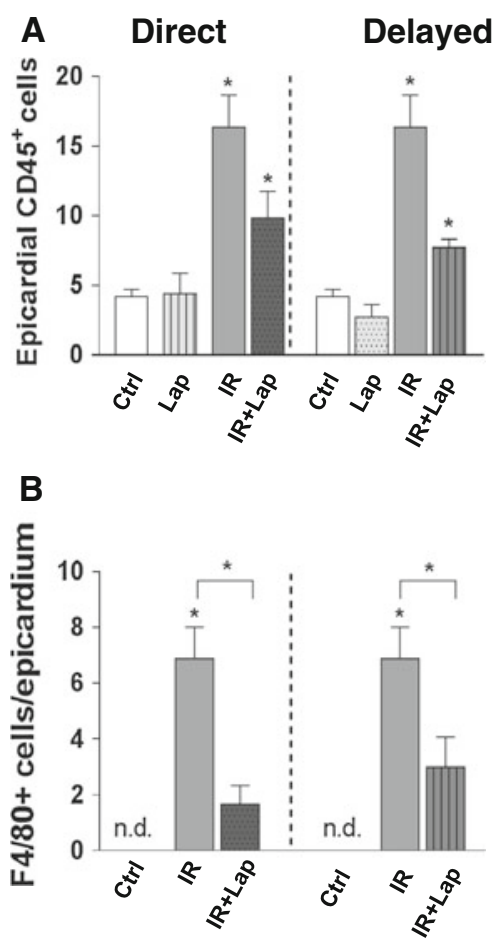

Fig. 5 Inflammatory changes after ErbB2 inhibition alone or in combination with irradiation. a Quantification of CD45+cells $\mathbf{b}$ and of F4/80+cells per section in the epicardium. Each value represents the mean $( \pm$ SEM) for minimal five mice per group. $* p<0.05$ compared to age-matched untreated controls. n.d. not determined. Ctrl control; Lap lapatinib; IR irradiation; IR+lap irradiation combined with lapatinib

or in combination with direct lapatinib. However, when delayed lapatinib was given to irradiated mice there was a trend for increased myocardial and blood vessel damage, including dilation of capillaries, degeneration of coronary arteries, degeneration of the myocardium, and hypertrophy of the myocardium (Fig. 7). No severe changes were detected in Dox-treated mice that survived until 40 weeks. Examination of prematurely sacrificed, sick animals did demonstrate damage but this group comprised only a few mice, sacrificed at various times after treatment, so no semiquantitative analysis was done. Electron microscopy (EM) analysis indicated small changes in myocyte morphology, like local vacuolization, after Dox or lapatinib treatment alone. Changes were enhanced when Dox was combined with lapatinib, either direct or delayed. Focal damage was seen with degenerative changes in the mitochondria, cloudyswollen phenotype and disorganized, disrupted Z-bands in some cardiomyocytes, but normal looking mitochondria and Z-bands in adjacent cells (Fig. 8).

\section{Lapatinib decreased left ventricle function}

Cardiac function tests, determined by gated SPECT/CT, showed increases in ESV after lapatinib alone (34 and
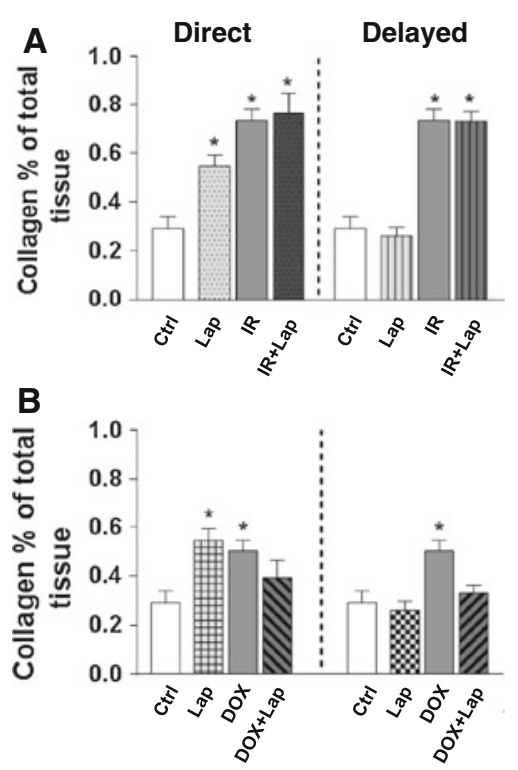

Fig. 6 Fibrotic changes after ErbB2 inhibition alone or in combination with irradiation or doxorubicin. a Collagen per unit area expressed as percentage of total tissue for animals treated with irradiation. b Collagen per unit area expressed as percentage of total tissue for animals treated with doxorubicin $* p<0.05$ compared to age-matched untreated controls. Each value represents the mean $( \pm$ SEM) for minimal five mice per group. n.d. not determined. Ctrl control; Lap lapatinib; DOX doxorubicin; DOX+Lap doxorubicin combined with lapatinib; IR irradiation; IR+lap irradiation combined with lapatinib

$16 \%$ increase for direct and delayed schedules) (Fig. 9). Similar increases in EDV were seen (11 and $9 \%$ for direct and delayed schedules) (data not shown). This resulted in decreased EF (22 and $9 \%$ decreases after direct and delayed lapatinib) (Fig. 9). PFR, maximum down slope of left-ventricular volume, also showed significant decrease after lapatinib treatment (20 and $21 \%$ after direct and delayed lapatinib) (Fig. 9). Neither 14 Gy nor $3 \times 4 \mathrm{mg} / \mathrm{kg}$ Dox induced significant changes in cardiac function, either given alone or in combination with lapatinib.

\section{Discussion}

In this study, we investigated the effect of direct and delayed inhibition of EGFR signaling combined with irradiation or Dox on cardiomyocyte survival, morphological damage, and heart function. We demonstrated that inhibiting ErbB2 in combination with irradiation or anthracyclines did not further decrease myocyte cell viability in vitro. Combined inhibition of ErbB1 and ErbB2 also did not enhance radiation or Dox induced cardiac damage in mice. Indeed, the radiation-induced inflammatory responses were inhibited by lapatinib. 

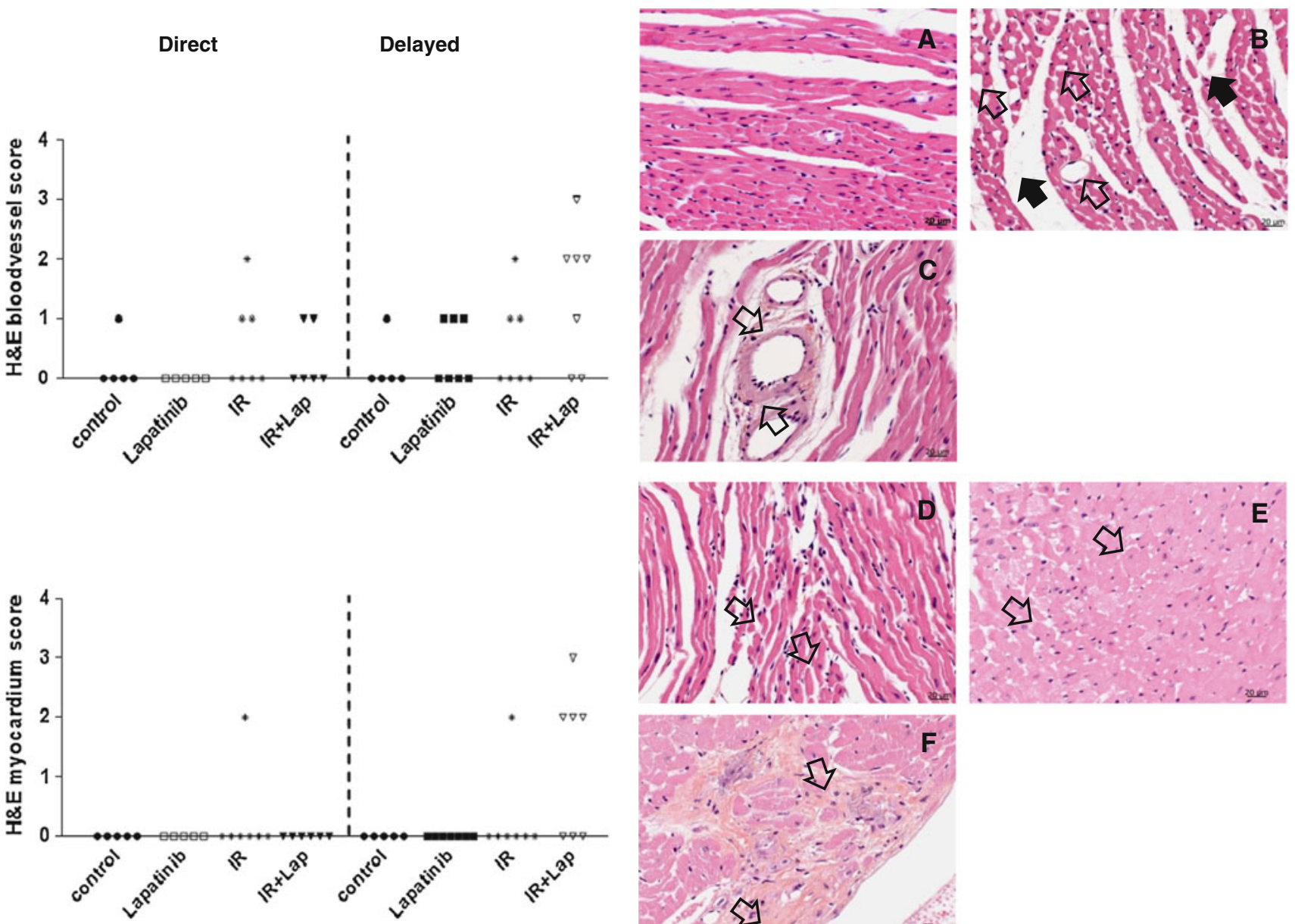

Fig. 7 Histological changes in the myocardium and blood vessels at 40 weeks after treatment with irradiation or doxorubicin alone or in combination with lapatinib. H\&E sections of the heart of mice that survived treatment with irradiation alone or in combination with lapatinib. Blood vessel and myocardium were scored for cardiac events at 40 weeks after treatment. Each symbol represents the score of one mouse (minimal five mice per group). a Age-matched control

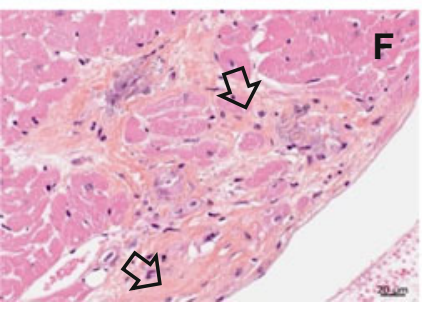

heart. b Dilated capillaries (open arrow) and edema in the stroma (dark arrow) of an irradiated heart. c Hyaline-like degeneration of the coronary arterioles (open arrows). d Atrophy of the myocardium (small, misshapen myocytes, and separated from each other, open arrows). (e) Degeneration of the myocardium. f Focal fibrosis (open arrows) and degeneration of endocardium

Inhibition of ErbB2 in HER2-overexpressing breast cancers became a standard (adjuvant) treatment as it results in improvement of outcome [18]. Often this is combined with anthracycline CT [19]. In addition, most patients receive adjuvant RT, either after breast conserving surgery or mastectomy [20]. Previous studies have shown an increase in cardiac events after combining RT with anthracyclines, such as decline in LVEF or congestive heart failure [11, 13, 21]. Long term follow up studies with ErbB2 inhibiting agents combined with these modalities are ongoing to determine both efficacy and safety, especially with regard to cardiac toxicity (ALTTO study; BIG 2-06/N063D).

EGFR signaling, including ligand NRG-1, plays an important role in adult cardiomyocyte survival, since it activates the pro-survival PI3K/pathway and stimulates cardiomyocyte maintenance and function [22]. Previous

studies in our lab, demonstrated the ability of the mouse heart to compensate functionally for significant structural damage after previous irradiation [17]. Compensatory mechanisms and pro-survial pathways might play a role in maintaining cardiomyocyte function in a damaged heart, at least until the extent of damage overwhelms the potential for stimulated survival. Sridharan et al. [16] recently demonstrated upregulation of ErbB signaling in the irradiated rat heart in parallel with developing cardiac fibrosis, leading to the suggestion that ErbB signaling and prosurvival pathways are activated in an attempt to regenerate the myocardium. Moreover, inactivation of ErbB4 in ventricular muscle cells led to a severe dilated cardiomyopathy, demonstrating the important role of ErbB signaling in the myocardium [23].

This raises the question of whether inhibition of these pathways could impair attempts of the damaged heart to 


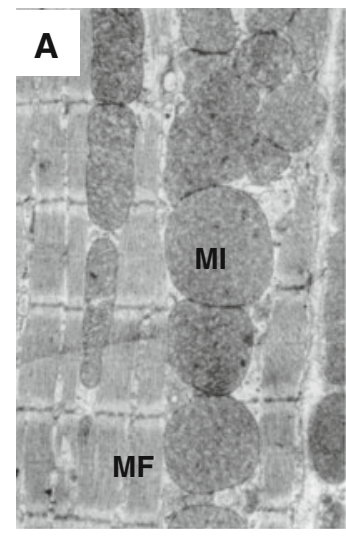

$600 \mathrm{~nm}$

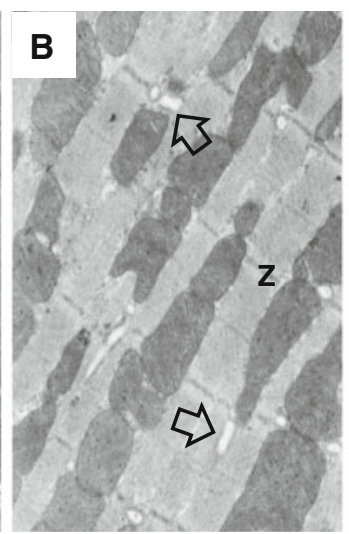

$300 \mathrm{~nm}$

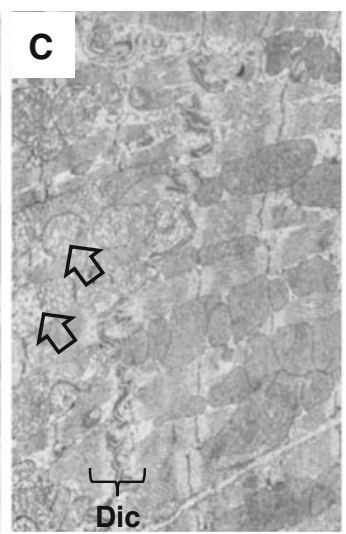

$500 \mathrm{~nm}$

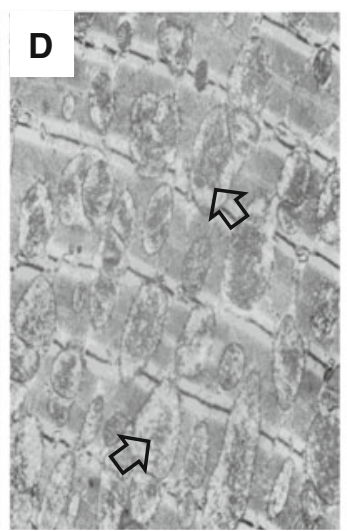

$500 \mathrm{~nm}$

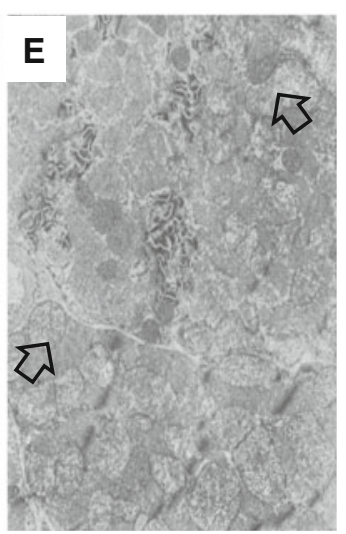

$500 \mathrm{~nm}$
Fig. 8 Mitochondrial changes in the myocardium after ErbB2 inhibition. Electron microscopic images of the myocardium of mice treated with lapatinib alone or in combination with doxorubicin at 40 weeks after treatment. a Age-matched control $(n=3)$; cardiomyocytes show organized sarcomeres characterized by parallel myofilaments anchored to $\mathrm{Z}$ bands and mitochondria were perfectly aligned and packed. b Doxorubicin treatment alone $(n=3)$; myofilament arranged regularly and mitochondria were aligned with focal vacuolization (arrow). c Lapatinib treatment alone $(n=3)$; focal damage per cardiomyocyte. Mitochondrial volume increased, mitochondrial cristae were fuzzy and had a cloudy swollen phenotype (arrow). d Direct lapatinib set up combined with doxorubicin $(n=3)$; e delayed lapatinib set up combined with doxorubicin $(n=3)$; disorganized mitochondria, mitochondrial cristae were fuzzy and had a cloudy swollen phenotype (arrow), increased volume of mitochondria. MI mitochondria; MF myofibril; Z z-bands; Dic Discus interculatis regenerate. Similar to the findings of Sridharan et al. [16], we found increased expression of both ErbB2 and ErbB4 in mouse hearts at 40 weeks after irradiation, in parallel with developing fibrosis (Supplementary Fig. 1). However, this upregulation was not inhibited in the presence of lapatinib. Our data also do not indicate cardiac dysfunction after radiation alone or in combination with lapatinib, in contrast to the mild but significant changes in cardiac function after lapatinib alone (Fig. 9). These data are consistent with the important role of ErbB signaling to maintain myocardium functionality and imply that that this compensatory mechanisms initiated by irradiation still operate in the presence of lapatinib.

Treatment of adult human cardiomyocytes, which have low expression of HER2, with trastuzumab, did not alter cell viability, nor did it enhance toxicity induced by either radiation or Dox (Fig. 2). This is consistent with the knowledge that anthracyclines cause acute irreversible damage to the cardiomyocytes through generation of free radicals and cardiomyocyte apoptosis [24], whereas trastuzumab-induced cardiac toxicity is dose-dependent and largely reversible [25]. The doses of trastuzumab we used were sufficient to cause significant reduction in viability in HER2-overexpressing tumor cells (data not shown), but the cardiomyocytes were less sensitive to ErbB2 inhibition. Some studies, using HER2-overexpressing breast cancer cells, have shown that inhibition of PI3K - pathway may be bypassed by lateral activation of other members of the HER family (Her3) [26, 27]. This raises the possibility, that activation of this compensatory feedback loop could thereby stimulate survival of cardiomyocytes.

Our in vitro results contrast somewhat with a recently published study by Hasinoff et al., in which pretreatment of isolated neonatal rat cardiac myocytes with HER1/HER2 inhibitor lapatinib potentiated doxorubicin-induced myocyte damage, assessed by LDH release and disrupted sarcomeres, although there was no enhancement of doxorubicin-induced apoptosis. A major difference between our study and that of Hasinoff is that they used neonatal cardiomyocytes and neuregulin is essential for the developing heart, which probably increases cardiomyocyte sensitivity to ErbB2 inhibition. In our study, we investigated the effects of trastuzumab (ErbB2 inhibitor) on adult cardiomyocyte survival after Dox or irradiation. We did not look at changes in cardiomyocyte function or morphology in vitro. However, our EM study from in vivo experiments would suggest enhanced cardiomyocyte damage from the combination of lapatinib and Dox, consistent with the data of Hasinoff.

Our findings suggest that trastuzumab or lapatinib induced cardiac toxicity acts by different mechanisms than anthracyclines or radiation. This raises the possibility that the cardiac toxicity seen in clinical studies where ErbB2 inhibition is combined with anthracyclines could be due to indirect effects, for example endothelial cell dysfunction and loss, with secondary damage to the cardiomyocytes.

We have previously shown [17] that local cardiac irradiation induces microvascular loss and damage. The current study shows that irradiation-induced endothelial 

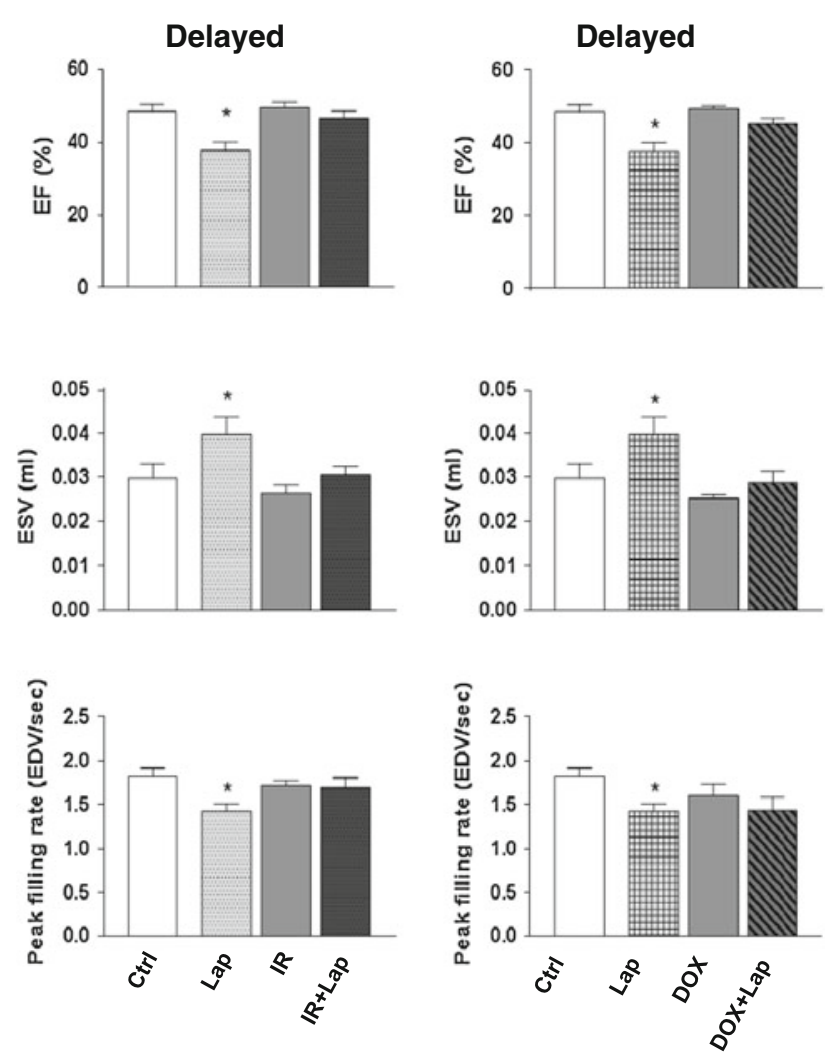

Fig. 9 Cardiac function (EF, ESV, and PFR) measured by gated SPECT at 40 weeks after treatment with irradiation or doxorubicin alone or in combination with lapatinib or sham treatment. Values represent mean \pm SEM (8-14 mice in each treatment group), ${ }^{*} p<0.05$ compared to age-matched untreated controls. Ctrl control; Lap lapatinib; DOX doxorubicin; DOX+Lap doxorubicin combined with lapatinib; IR irradiation; IR + lap irradiation combined with lapatinib

dysfunction and loss was not further enhanced by lapatinib, either when given at the time of irradiation (direct) or delayed for 20 weeks. Mechanisms of radiation-induced cardiac toxicity include activation of TGF $\beta$ and production of chemokines and pro-inflammatory cytokines, which are largely independent of the PI3K-pathway determining cardiomyocyte survival. However, the MAPK pathway has been implicated in regulation of permeability in endothelial cells [28], something we found to be increased by irradiation. These observations are consistent with the concept that mechanisms of radiation-induced cardiac damage do not involve ErbB signaling.

Slow turnover tissues, like the myocardium, have a low proliferative capacity and a small stem-cell component, and therefore have a limited ability to repair damage. Radiationinduced cardiac toxicity is initiated by an acute inflammatory response, which progresses to fibrosis without proper tissue regeneration [29]. We found that the inflammatory response initially activated by irradiation was decreased by lapatinib. We can speculate that, since the MAPK pathway mediates myocardial pro-inflammatory cytokine production, inhibiting MAPK pathway with lapatinib may decrease the pro-inflammatory cytokine production $[30,31]$. However, we have no data to support this hypothesis.

A further consequence of irradiation is the development of fibrosis. Similar to previous studies, our study showed increased cardiac fibrosis after irradiation or Dox alone. However, this was not further enhanced by lapatinib. On the other hand, 20 weeks of lapatinib alone in the direct set up induced fibrosis in mice sacrificed at 40 weeks. This is in contrast to no increase in fibrosis when lapatinib was delayed until 20 weeks and continued until sacrifice at 40 weeks. This might be explained by the delayed fibrotic response of the myocardium, which required time to develop after cessation of the lapatinib. We did not evaluate mice at later follow-up times than 40 weeks.

Changes in the microvasculature after Dox alone or with lapatinib were not seen. Nevertheless, fibrosis and degenerated cardiomyocytes (EM study) and 27-36\% animal lethality, as well as decreased cell viability in vitro, do indicate doxorubicin-induced damage to the myocardium via cardiomyocytes. Although anthracyclines induce acute damage, clinical symptoms are mostly detectable months or years after treatment [32]. Therefore, besides acute myocardial damage, late and indirect microvascular damage may occur at later times.

We conclude that ErbB2 inhibition did not enhance direct radiation or anthracyclines toxicity of cardiomyocytes in vitro. Radiation-induced microvascular damage was not further enhanced by ErbB2 inhibition and the inflammatory response was decreased. Myocardial damage was induced by anthracyclines but not further enhanced by ErbB2 inhibition. Treatment of mice with the ErbB 1/2blocking agent lapatinib did not further enhance the risk of radiation or anthracycline-induced cardiac toxicity up to 40 weeks after treatment.

Acknowledgments The authors would like to thank Bert Pool, Department of Nuclear Medicine, The Netherlands Cancer Institute, for help with the NanoSPECT/CT and Dr. Jack Cleutjens, Department of Pathology, University of Maastricht, for help with the Leica Qwin morphometry system. Our thanks go to Hans Janssen and Nico Ong, Division of Cell Biology, The Netherlands Cancer Institute, for their expertise in electron microscopy. Moreover, we would like to thank Antsje Nolles and Sjoers van der Horst for their technical assistance regarding in vitro experiments and real-time PCR analysis.

Conflict of interest The authors declare that they have no conflict of interest.

Open Access This article is distributed under the terms of the Creative Commons Attribution Noncommercial License which permits any noncommercial use, distribution, and reproduction in any medium, provided the original author(s) and the source are credited. 


\section{References}

1. Bovelli D, Plataniotis G, Roila F (2010) Cardiotoxicity of chemotherapeutic agents and radiotherapy-related heart disease: ESMO clinical practice guidelines. Ann Oncol 21(Suppl 5):v277v282

2. Marks LB, Yu X, Prosnitz RG, Zhou SM, Hardenbergh PH et al (2005) The incidence and functional consequences of RT-associated cardiac perfusion defects. Int $\mathbf{J}$ Radiat Oncol Biol Phys 63:214-223

3. Prosnitz RG, Hubbs JL, Evans ES, Zhou SM, Yu X et al (2007) Prospective assessment of radiotherapy-associated cardiac toxicity in breast cancer patients: analysis of data 3 to 6 years after treatment. Cancer 110:1840-1850

4. Lind PA, Pagnanelli R, Marks LB, Borges-Neto S, Hu C et al (2003) Myocardial perfusion changes in patients irradiated for left-sided breast cancer and correlation with coronary artery distribution. Int J Radiat Oncol Biol Phys 55:914-920

5. Nahta R, Hortobagyi GN, Esteva FJ (2003) Growth factor receptors in breast cancer: potential for therapeutic intervention. Oncologist 8:5-17

6. Allred DC, Clark GM, Tandon AK, Molina R, Tormey DC et al (1992) HER-2/neu in node-negative breast cancer: prognostic significance of overexpression influenced by the presence of in situ carcinoma. J Clin Oncol 10:599-605

7. Xue C, Wyckoff J, Liang F, Sidani M, Violini S et al (2006) Epidermal growth factor receptor overexpression results in increased tumor cell motility in vivo coordinately with enhanced intravasation and metastasis. Cancer Res 66:192-197

8. Vogel CL, Cobleigh MA, Tripathy D, Gutheil JC, Harris LN et al (2002) Efficacy and safety of trastuzumab as a single agent in first-line treatment of HER2-overexpressing metastatic breast cancer. J Clin Oncol 20:719-726

9. Tan M, Yu D (2007) Molecular mechanisms of erbB2-mediated breast cancer chemoresistance. Adv Exp Med Biol 608:119-129

10. Joensuu H, Kellokumpu-Lehtinen PL, Bono P, Alanko T, Kataja $\mathrm{V}$ et al (2006) Adjuvant docetaxel or vinorelbine with or without trastuzumab for breast cancer. N Engl J Med 354:809-820

11. Romond EH, Perez EA, Bryant J, Suman VJ, Geyer CE Jr et al (2005) Trastuzumab plus adjuvant chemotherapy for operable HER2-positive breast cancer. N Engl J Med 353:1673-1684

12. Feldman AM, Lorell BH, Reis SE (2000) Trastuzumab in the treatment of metastatic breast cancer : anticancer therapy versus cardiotoxicity. Circulation 102:272-274

13. Perez EA, Koehler M, Byrne J, Preston AJ, Rappold E et al (2008) Cardiac safety of lapatinib: pooled analysis of 3689 patients enrolled in clinical trials. Mayo Clin Proc 83:679-686

14. De Keulenaer GW, Doggen K, Lemmens K (2010) The vulnerability of the heart as a pluricellular paracrine organ: lessons from unexpected triggers of heart failure in targeted ErbB2 anticancer therapy. Circ Res 106:35-46

15. Zhao YY, Sawyer DR, Baliga RR, Opel DJ, Han X et al (1998) Neuregulins promote survival and growth of cardiac myocytes. Persistence of ErbB2 and ErbB4 expression in neonatal and adult ventricular myocytes. J Biol Chem 273:10261-10269

16. Sridharan V, Sharma SK, Moros EG, Corry PM, Tripathi P et al (2013) Effects of radiation on the epidermal growth factor receptor pathway in the heart. Int J Radiat Biol 89(7):539-547

17. Seemann I, Gabriels K, Visser NL, Hoving S, te Poele JA et al (2012) Irradiation induced modest changes in murine cardiac function despite progressive structural damage to the myocardium and microvasculature. Radiother Oncol 103:143-150

18. Mitri Z, Constantine T, O'Regan R (2012) The HER2 receptor in breast cancer: pathophysiology, clinical use, and new advances in therapy. Chemother Res Pract 2012:743193

19. Slamon DJ, Leyland-Jones B, Shak S, Fuchs H, Paton V et al (2001) Use of chemotherapy plus a monoclonal antibody against HER2 for metastatic breast cancer that overexpresses HER2. N Engl J Med 344:783-792

20. Halyard MY, Pisansky TM, Dueck AC, Suman V, Pierce L et al (2009) Radiotherapy and adjuvant trastuzumab in operable breast cancer: tolerability and adverse event data from the NCCTG Phase III Trial N9831. J Clin Oncol 27:2638-2644

21. Tan-Chiu E, Yothers G, Romond E, Geyer CE Jr, Ewer M et al (2005) Assessment of cardiac dysfunction in a randomized trial comparing doxorubicin and cyclophosphamide followed by paclitaxel, with or without trastuzumab as adjuvant therapy in nodepositive, human epidermal growth factor receptor 2-overexpressing breast cancer: NSABP B-31. J Clin Oncol 23:7811-7819

22. Lemmens K, Doggen K, De Keulenaer GW (2007) Role of neuregulin-1/ErbB signaling in cardiovascular physiology and disease: implications for therapy of heart failure. Circulation 116:954-960

23. Garcia-Rivello H, Taranda J, Said M, Cabeza-Meckert P, VilaPetroff $M$ et al (2005) Dilated cardiomyopathy in Erb-b4-deficient ventricular muscle. Am J Physiol Heart Circ Physiol 289:H1153-H1160

24. Doroshow JH (1983) Effect of anthracycline antibiotics on oxygen radical formation in rat heart. Cancer Res 43:460-472

25. Ewer MS, Vooletich MT, Durand JB, Woods ML, Davis JR et al (2005) Reversibility of trastuzumab-related cardiotoxicity: new insights based on clinical course and response to medical treatment. J Clin Oncol 23:7820-7826

26. Garrett JT, Olivares MG, Rinehart C, Granja-Ingram ND, Sanchez V et al (2011) Transcriptional and posttranslational upregulation of HER3 (ErbB3) compensates for inhibition of the HER2 tyrosine kinase. Proc Natl Acad Sci USA 108:5021-5026

27. Serra V, Scaltriti M, Prudkin L, Eichhorn PJ, Ibrahim YH et al (2011) PI3K inhibition results in enhanced HER signaling and acquired ERK dependency in HER2-overexpressing breast cancer. Oncogene 30:2547-2557

28. Kevil CG, Oshima T, Alexander B, Coe LL, Alexander JS (2000) $\mathrm{H}(2) \mathrm{O}(2)$-mediated permeability: role of MAPK and occludin. Am J Physiol Cell Physiol 279:C21-C30

29. Brush J, Lipnick SL, Phillips T, Sitko J, McDonald JT et al (2007) Molecular mechanisms of late normal tissue injury. Semin Radiat Oncol 17:121-130

30. Craig R, Larkin A, Mingo AM, Thuerauf DJ, Andrews C et al (2000) p38 MAPK and NF-kappa B collaborate to induce interleukin-6 gene expression and release. Evidence for a cytoprotective autocrine signaling pathway in a cardiac myocyte model system. J Biol Chem 275:23814-23824

31. Meldrum, Dinarello CA, Cleveland JC, Cain BS, Shames BD et al (1998) Hydrogen peroxide induces tumor necrosis factor alphamediated cardiac injury by a P38 mitogen-activated protein kinasedependent mechanism. Surgery 124:291-296 discussion 297

32. Swain SM (2008) Cardiovascular complications of breast cancer therapy. Clin Adv Hematol Oncol 6(247-248):282 